\title{
Comparison between Theoretical and Experimental Radial Electron Temperature Profiles in a Low Density Weakly Ionized Plasma
}

\author{
Ahmed Rida Galaly ${ }^{1,2 *}$, Guido Van Oost ${ }^{3,4,5}$ \\ ${ }^{1}$ Department of Physics, Faculty of Science, Beni-Suef University, Beni Suef, Egypt \\ ${ }^{2}$ Department of Engineering Science, Faculty of Community, Umm Al_Qura University, Mecca, Mecca Province, KSA \\ ${ }^{3}$ Department of Applied Physics, Ghent University, Technicum B4, Ghent, Belgium \\ ${ }^{4}$ National Research Nuclear University "MEPHI”, Moscow, Russia \\ ${ }^{5}$ National Research University “Moscow Power Engineering Institute”, Moscow, Russia \\ Email: ^ahmed_galaly@yahoo.com
}

How to cite this paper: Galaly, A.R. and Van Oost, G. (2019) Comparison between Theoretical and Experimental Radial Electron Temperature Profiles in a Low Density Weakly Ionized Plasma. Journal of Modern Physics, 10, 699-716.

https://doi.org/10.4236/jmp.2019.107050

Received: May 15, 2019

Accepted: June 11, 2019

Published: June 14, 2019

Copyright $\odot 2019$ by author(s) and Scientific Research Publishing Inc. This work is licensed under the Creative Commons Attribution International License (CC BY 4.0).

http://creativecommons.org/licenses/by/4.0/ CC) (i) Open Access

\begin{abstract}
Experimental and theoretical studies of the radial distribution function of the electron temperature (RDFT) in a low-density plasma and weakly ionized gas for the abnormal glow region are presented. Experimentally, the electron temperatures and densities are measured by a Langmuir probe moved radially from the center to the edge of the cathode electrode for helium gas at different pressures in the low-pressure glow discharge. The comparison of the final experimental data for the radial distribution of electron temperatures and densities for different low pressures ranging from 0.2 to 1.2 torr, with the final proved equation of RDFT confirms that the electron temperatures decrease with increasing product of radial distance and gas pressures, showing a radial decrement dependence of the electron temperature from the center to the edge of the electrode. This is attributed to the increase of the number of electron-atom collisions at higher gas pressures and consequently of the rate of ionization. For the axial distance $(L)$ from the tip of the probe to cathode electrode and the cathode electrode radius $(R)$, a theoretical and experimental comparison for the two conditions $L<R$ and $L>R$, for both cases the produced plasma temperatures decrease and densities increase. It is concluded that the RDFT accurately shows a dramatic decrease for $L<R$ by $60 \%$ less than RDFT values for $L>R$ similar as for conditions of magnetized and unmagnetized effect for DC plasma. This means that the rate of plasma loss by diffusion decreased for $L<R$, agrees well with the applied of magnetic
\end{abstract}


field behavior.

\section{Keywords}

Radial Distribution, Electron Temperature, Ambipolar Diffusion, Low Pressure, Weakly Ionized Plasma

\section{Introduction}

Plasma is the fourth state of matter, considered to be a quasi-neutral medium. However, when a diagnostic such as probe (a small metallic electrode) is inserted into a weakly ionized plasma, a very thin sheath is formed around the conducting surface of the probe due to the redistribution of charges. The amplitude and the polarity of the probe potential control the motion of electrons and ions near the probe. When the probe potential is sufficiently negative, only the ions can reach the probe surface. The probe current is thus equal to the ion random current $\left(I_{r i}\right)$ [1] [2] [3].

Ambipolar diffusion is the most important process taking place within a weakly ionized plasma and considered as a vital process for the distribution of the plasma parameters. Assuming a Maxwellian velocity distribution [4], the plasma parameters especially electron temperature and electron density can be determined from the current-voltage characteristic curves of the probe [5]. Diffusion phenomena occur in a plasma when a spatial gradient of the charged species is present [6]. Furthermore, it is caused by the differently charged species having different diffusivities, hence there is a loss of neutrality [7] due to plasma rapidly diffusion for some of charged species more than the others. Furthermore, a minor loss of neutrality, however, induces an ambipolar electric field which, if the Debye length is sufficiently small, slows down the fast-diffusing species and speeds up the slow-diffusing species in such a way that the plasma remains quasi-neutral [8].

The experimental and numerical radial distribution of electron temperature and density from the axis of the tube up to the tube wall have been amply investigated [9] [10], but not the theoretical derivation in terms of the effect of Schottky condition and ambipolar diffusion due to the recurrence relation of the Bessel condition.

In the present work, an experimental study of the radial dependence of the electron temperature in a low-density plasma using a single probe as diagnostic technique was performed. Theoretically, it was proven that the ambipolar diffusion is an important factor in the weakly-ionized gas. Furthermore, a theoretical physical model for the radial distribution of the temperature was derived. The theoretical considerations are compatible with the experimental data of the temperatures at different pressures in a low-pressure glow discharge of a DC (cold cathode) magnetron sputtering unit. 


\section{Experimental Study}

\subsection{Experimental Set-Up}

Figure 1 shows the experimental setup of DC (cold cathode) magnetron sputtering unit to generate a glow discharge in a glass tube between two circular, parallel and movable metallic discs acting as electrodes. Two parallel electrodes made of aluminum, are enclosed in the discharge cell, one of the two electrodes is grounded represented cathode electrode and the other is movable to change the axial distance represented the anode electrode, where the axial distance $(L)$ between tip of the probe and the cathode electrode, $5 \mathrm{~cm}$ and with $3 \mathrm{~cm}$ in radius ( $R$ ) taking into account that we will deal with two conditions $R>L$ and $R<L$. The discharge unit is evacuated using a rotary pump to a base pressure of 7 mtorr. A pressure gauge is connected to the discharge tube to measure the inside gas pressure. A stationary DC-glow discharge was generated between two both electrodes, for different parameters as shown in Table 1.

Figure 1 also shows the schematic diagram of the spherical single Langmuir probe circuit. The probe made of molybdenum wire (diameter $3.0-\mathrm{mm}$ and length $0.5 \mathrm{~mm}$ ) and the tip of a probe is inside the glow discharge plasma. The single probe (between the cathode fixed at the ground potential, and the anode) move axially to measure the axial potential distribution between the two electrodes. Also, it moves radially from the center to the edge of the cathode electrode, and a potential $V_{P}$ is applied to the probe.

\subsection{Experimental Study of Axial Potential and Electric Field Distribution Measurements}

The potential distribution was measured at discharge currents of $10 \mathrm{~mA}$ and gas pressure of (0.2 - 1.2 torr) for He as shown in Figure 2, shows that the potential

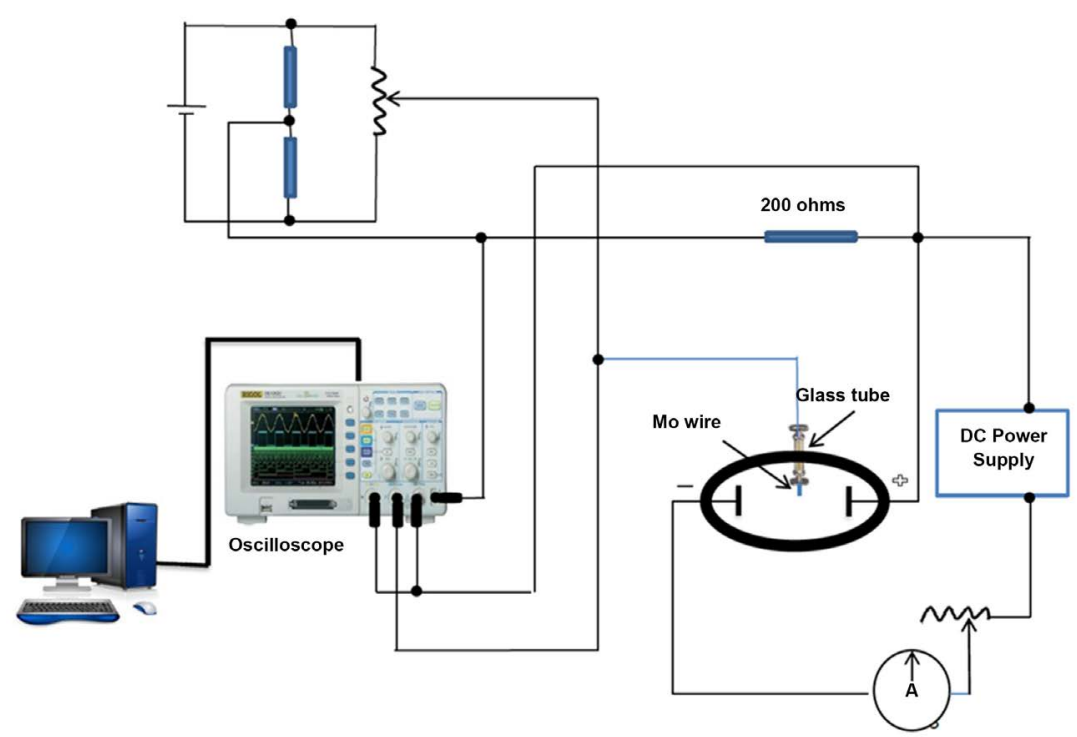

Figure 1. Schematic diagram of the experimental setup and the single Langmuir probe circuit. 


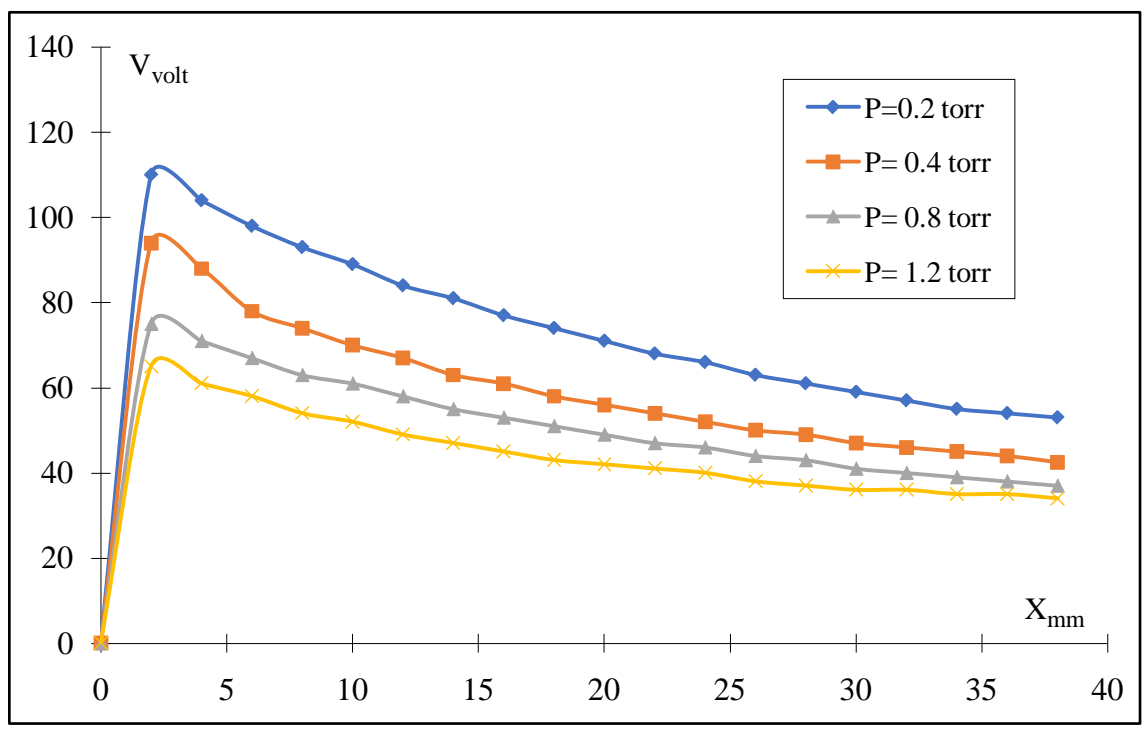

Figure 2. Axial potential distribution at constant current $(I=10 \mathrm{~mA})$.

Table 1. Shows the values of operating parameters.

\begin{tabular}{cc}
\hline Parameters & Values \\
\hline Discharge Current $\left(I_{a}\right)$ & $4-30 \mathrm{~mA}$ \\
Gas Pressure $(P)$ & $0.1-1.5 \mathrm{torr}$ \\
Discharge Voltage $\left(V_{a}\right)$ & $200-1200 \mathrm{~V} \mathrm{DC}$ \\
Current Density & $2-15 \mathrm{~mA} / \mathrm{m}^{2}$ \\
Working Gas & $\mathrm{He}$ \\
\hline
\end{tabular}

distribution can be divided into three regions. In region I (AB) (cathode fall), the potential increases sharply within a small discharge length. Whenever, gas breakdown takes place in the tube, a rapid growth in the rate of ionization, near the cathode is detected. Meanwhile the electrons transfer in the electric field much more faster than positive ions (due to their masses), electrons are swept rapidly towards the anode leaving a dense positive space charge near the cathode. Thus, the electric field is distorted and most of the applied potential is dropped across a narrow space in front of the cathode. Region II (BC) (negative glow) indicates that the potential decreases slightly and hence the electric field will be weak, since this region contains many free electrons. In region III (CD) (positive column), the potential distribution is nearly constant and linear since the positive and negative carriers densities are closely, equal. The positive column can be extended to any length to fill the remaining space between the end of the negative glow and the anode.

Values of the electric field distribution are obtained by differentiating the measured potential distribution of Figure 2. Figure 3 and Figure 4 show the electric field distribution for He discharge. In the cathode fall region at edge and center, high electric field is observed which is decreased sharply away from the 


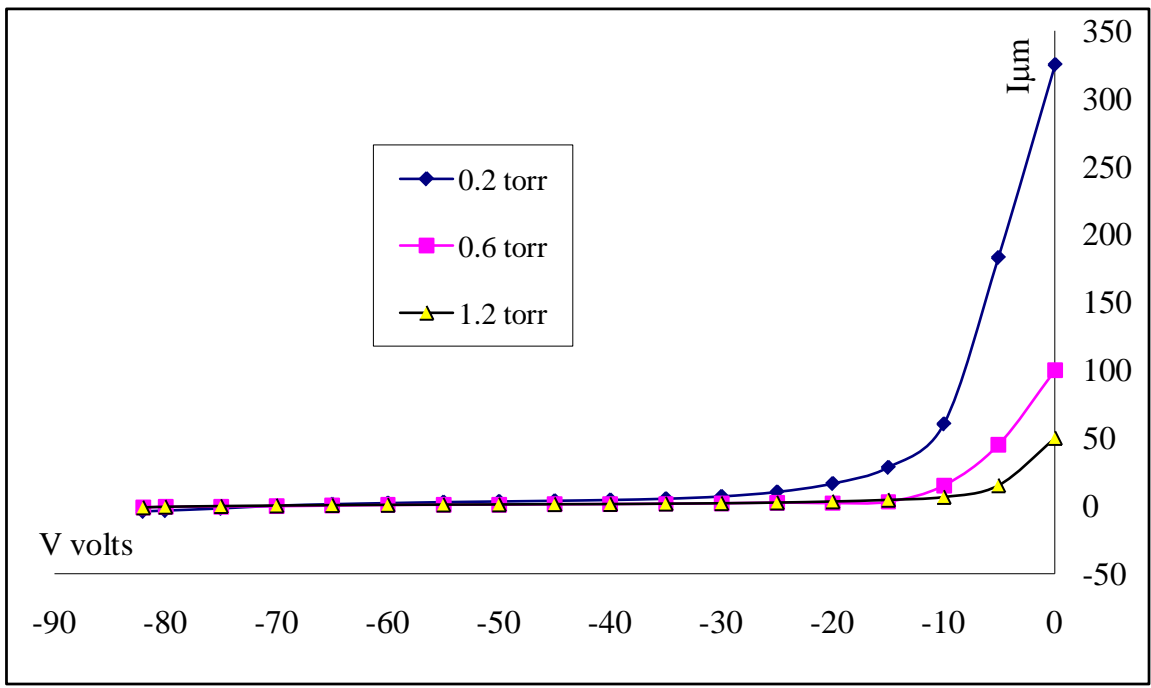

Figure 3. I-V curves of the single probe for cathode fall region at different He pressures at the edge.

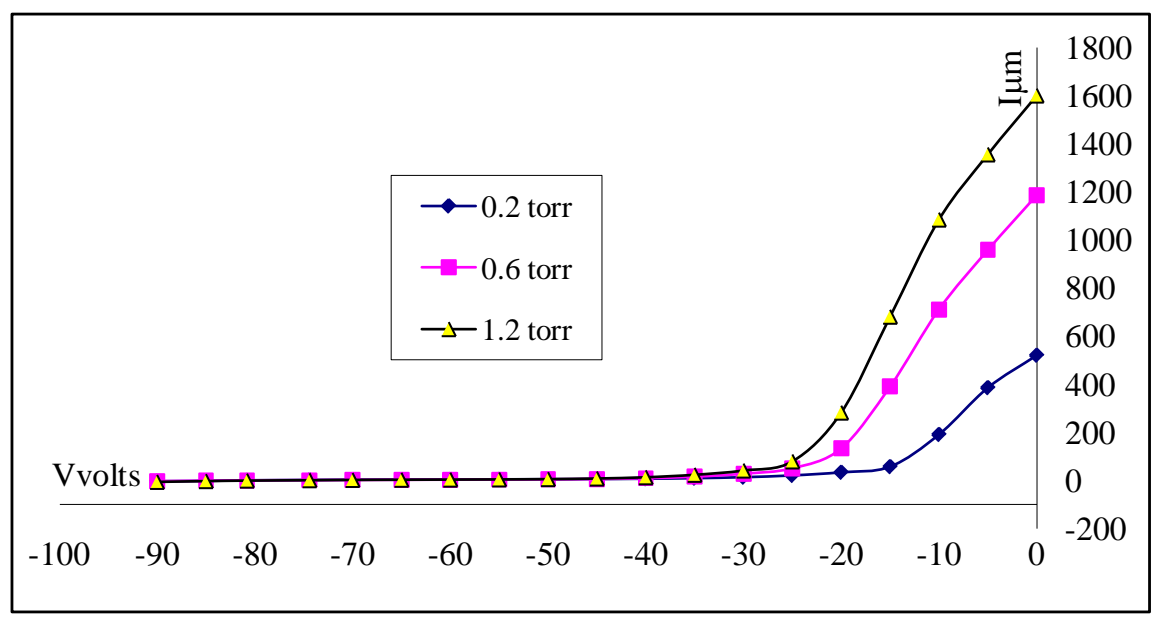

Figure 4. Measured I-V curves of the single probe for cathode fall region at different $\mathrm{He}$ pressures at the center.

cathode. This is related to the intense positive space charge which lies in front of the cathode. This acts as an accelerator for the electrons towards anode. Thus, the electrons emitted from the cathode are then accelerated away, until they reach the negative glow region where the electric field becomes weak (zero and sometimes it reaches a negative value). In this region, the gained kinetic energy of the electrons is dissipated in collisions with the atoms of the gas and thus secondary electrons would be produced. In the positive column region constant and linear electric field is needed to maintain discharge along the large length of the column which is required to carry the discharge current.

\subsection{Experimental Study of Radial Dependence of the Electron Temperature}

The region investigated in the present work is the abnormal region. The radial 
dependence of the electron temperature in low-density plasma at edge and center of the cathode electrode has been studied for different He pressures.

The plasma parameters like the electron temperature and electron density can be determined from the current-voltage (I-V) characteristic curve of the single Langmuir probe, based on the theory and the fundamental technique discussed in detail in many articles [10] [11].

Figure 3 and Figure 4 show I-V characteristic curves of the single probe at different He pressures at edge and center of the cathode electrode for the abnormal glow region [12]. The probe was moved to investigate the radial distribution from the center to the edge of the cathode, in the direction perpendicular to the direction of the electric field lines. Figure 5 and Figure 6 show the radial temperatures and densities distributions at different pressures from center to the edge. $T_{e}$ is decreased and $\mathrm{n}_{\mathrm{e}}$ increased due to the general trend that values of $T_{e}$ and $n_{e}$ are inversely proportional [13].

Increasing the helium working pressures from 0.2 to 1.2 torr, the temperature $T_{e}$ decreases at the center from $9.7 \mathrm{eV}$ to $5.5 \mathrm{eV}$ and at the edge from 4.1 to 1.15 $\mathrm{eV}$. Values of densities $\mathrm{n}_{\mathrm{e}}$ increased at center $\mathrm{n}_{\mathrm{e}}$ from $0.6 \times 10^{9} \mathrm{~cm}^{-3}$ to $1.3 \times 10^{9}$ $\mathrm{cm}^{-3}$ and at the edge from 2.5 to $3.95 \times 10^{9} \mathrm{~cm}^{-3}$.

\section{Theoretical Consideration of the Radial Dependence of the Electron Temperature}

Conductivity at the tube axis, and charge density of plasma according to the following expression [14] [15]

$$
D_{s}=D_{a}\left[1-\mu_{e} \frac{\rho}{\sigma}\right]
$$

where $D_{a}$ is the ambipolar diffusion coefficient as follows:

The flow of ions and electrons are the same, hence

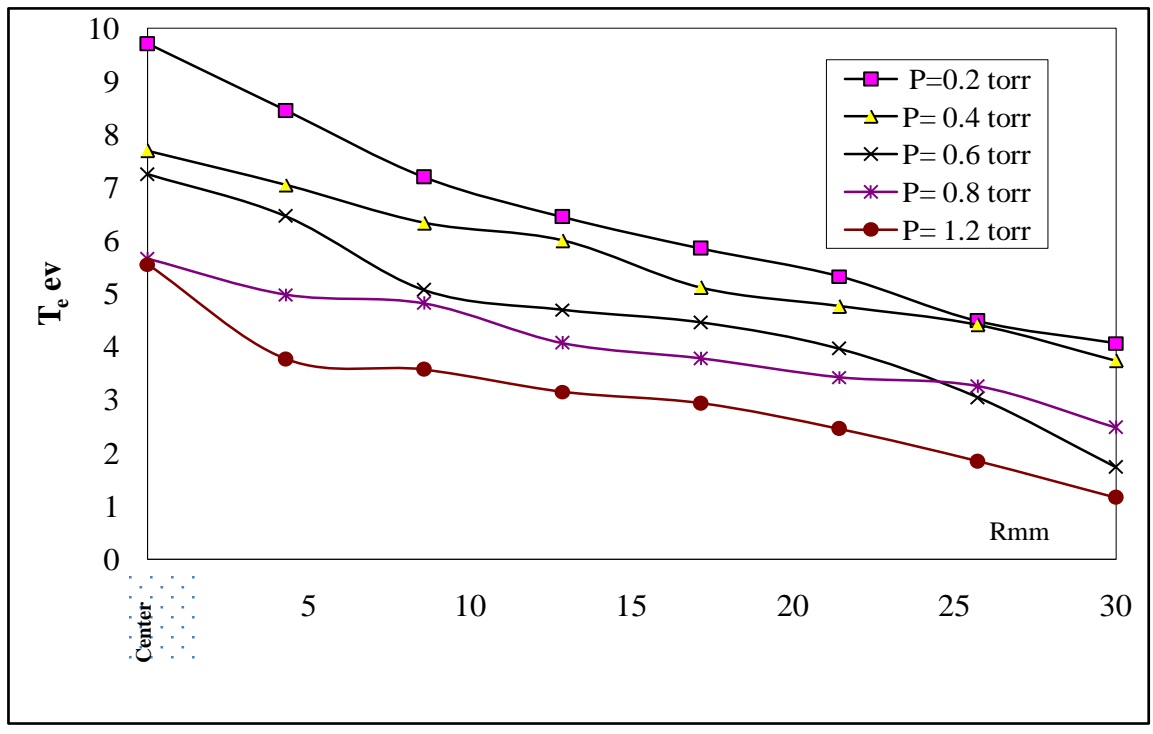

Figure 5. The Experimental radial temperature distribution of abnormal region for helium at different pressures. 


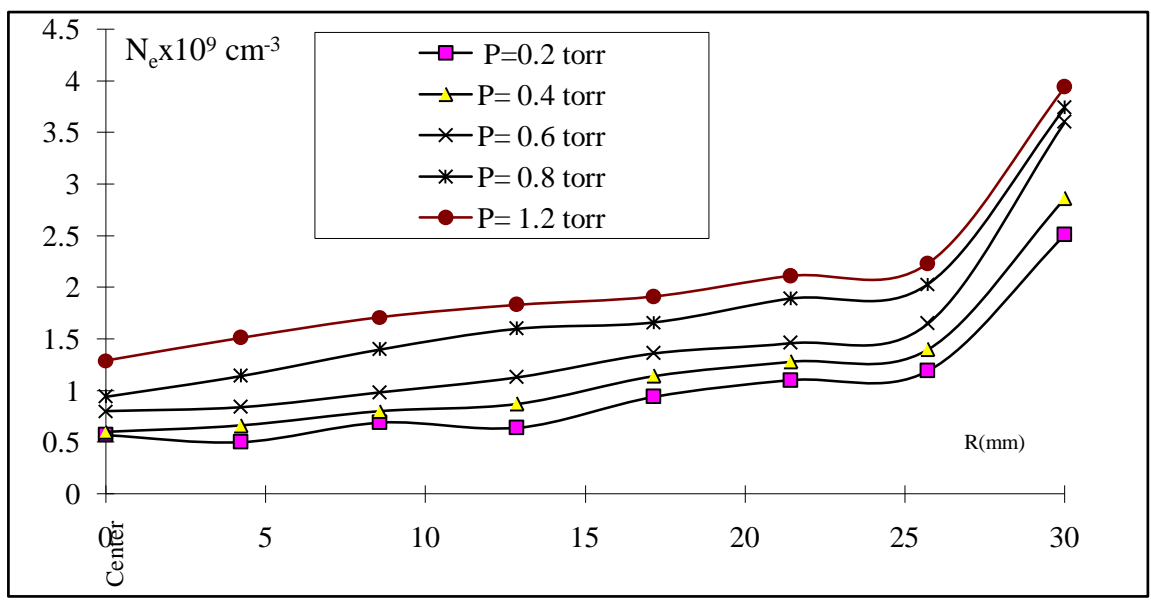

Figure 6. Measured radial density distribution of abnormal region for helium at different pressures.

$$
\Gamma=\Gamma_{i}=\Gamma_{e}
$$

(Congruence approximation). If there is no external electric field, the fluxes of ions and electrons (drift-diffusion model) can be expressed as:

$$
\begin{gathered}
\Gamma_{i}=-D_{i} \nabla n_{i}+\mu_{i} n_{i} E_{D} \\
\Gamma_{e}=-D_{e} \nabla n_{e}+\mu_{e} n_{e} E_{D}
\end{gathered}
$$

where $E_{D}$ follows Poisson's equation. If we multiply the first equation by $\mu_{e} n_{e}$ and the second one by $\mu_{i} n_{i}$ and then subtract them, we get:

$$
\mu_{i} n_{i} \Gamma_{e}-\mu_{e} n_{e} \Gamma_{i}=\mu_{e} n_{e} D_{i} \nabla n_{i}-\mu_{e} n_{e} \mu_{i} n_{i} E_{D}-\mu_{i} n_{i} D_{e} \nabla n_{e}+\mu_{i} n_{i} \mu_{e} n_{e} E_{D}
$$

Since $\Gamma=\Gamma_{i}=\Gamma_{e}$, the terms with $E_{D}$ cancel each other out and using $n_{e}=n_{i}$, the expression for the flow of particles becomes:

$$
\Gamma=\frac{-\left(D_{e} \mu_{i}-D_{i} \mu_{e}\right)}{\mu_{i}-\mu_{e}} \nabla n_{i}
$$

With the ambipolar diffusion coefficient being

$$
D_{a}=\frac{D_{e} \mu_{+}-D_{+} \mu_{e}}{\mu_{+}-\mu_{e}}
$$

and $\rho$ is the charge density in the plasma given by:

$$
\rho=e\left[n_{+}-n_{e}\right]
$$

and $\sigma$ is the conductivity at the tube axis given by:

$$
\sigma=e\left[\mu_{+} n_{+}-\mu_{e} n_{e}\right]
$$

Considering that diffusion coefficient $D$ given by:

$$
D=\frac{k T}{m v} \mathrm{~m} / \mathrm{s}^{2}
$$

where $\mu_{\varepsilon,+}$ are the mobility of electrons (e) and ions (+), $k T(\mathrm{ev})$ is the particle temperature, $v$ is the collision frequency between electrons and neutral atoms in Hz. By substituting with Equations (2, 3 and 4) into Equation (1), then we get 


$$
D_{s}=\frac{D_{e} \mu_{+}-D_{+} \mu_{e}}{\mu_{+}-\mu_{e}}\left[1-\mu_{e} \frac{n_{+}-n_{e}}{\mu_{+} n_{+}-\mu_{e} n_{e}}\right]
$$

But by substituting by the Einstein relation [16] [17]:

$$
\mu=\frac{\lceil q\rceil D}{k T}
$$

into Equation (6) by the value of D, then we get

$$
D_{s}=\frac{\mu_{e} \mu_{+} K T_{e}-\mu_{e} \mu_{+} K T_{+}}{q\left(\mu_{+}-\mu_{e}\right)}\left[1-\mu_{e} \frac{n_{+}-n_{e}}{\mu_{+} n_{+}-\mu_{e} n_{e}}\right]
$$

or

$$
D_{s}=\frac{\mu_{e} \mu_{+}\left(K T_{e}-K T_{+}\right)}{e\left(\mu_{+}-\mu_{e}\right)}\left[\frac{\mu_{+} n_{+}-\mu_{e} n_{e}-\mu_{e} n_{+}+\mu_{e} n_{e}}{\mu_{+} n_{+}-\mu_{e} n_{e}}\right]
$$

Neglecting $k T_{+}$, where $k T_{e} \gg k T_{+}$finally results in

Then

$$
\begin{aligned}
& D_{s}=\frac{n_{+} \mu_{e} \mu_{+}\left(k T_{e}\right)}{e\left(\mu_{+}-\mu_{e}\right)}\left[\frac{\mu_{+}-\mu_{e}}{\mu_{+} n_{+}-\mu_{e} n_{e}}\right] \\
& D_{s}=\frac{n_{+} \mu_{e} \mu_{+}\left(k T_{e}\right)}{e}\left[\frac{1}{\mu_{+} n_{+}-\mu_{e} n_{e}}\right]
\end{aligned}
$$

Then

$$
D_{s}=\frac{k T_{e}}{e}\left[\frac{\mu_{e} \mu_{+}}{\mu_{+}-\mu_{e} \frac{n_{e}}{n_{+}}}\right]
$$

or

$$
D_{s}=\frac{k T_{e}}{e}\left[\frac{\mu_{e}}{1-\frac{\mu_{e}}{\mu_{+}} \frac{n_{e}}{n_{+}}}\right]
$$

Substituting $\mu_{e}=\frac{e}{m_{e} v_{e}}$ and $\mu_{i}=\frac{e}{m_{i} v_{i}}$ from Equations (5) and (7) into Equation (12) results in

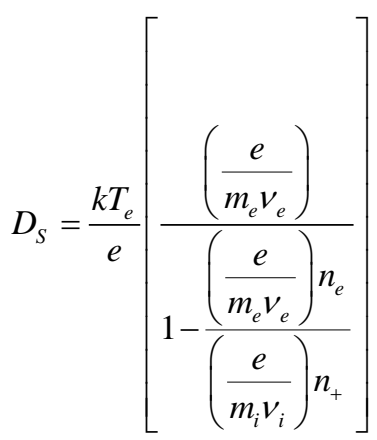

or 


$$
D_{S}=k T_{e}\left[\frac{\frac{1}{m_{e} v_{e}}}{1-\frac{m_{i} v_{i} n_{e}}{m_{e} v_{e} n_{+}}}\right]
$$

or

$$
\begin{gathered}
D_{S}=k T_{e}\left[\frac{n_{+}}{m_{e} v_{e} n_{+}-m_{i} v_{i} n_{e}}\right] \\
\frac{D_{S}}{v_{i}}=\frac{k T_{e}}{m_{e} v_{e} v_{i}-\frac{m_{i} v_{i}^{2} n_{e}}{n_{+}}}
\end{gathered}
$$

Experimentally, due to the diffusion process during plasma formation an interesting process occur in the plasma formation stage of the basil discharge. Theoretically, basil discharge process related with the recurrence relation for Bessel condition as shown in appendix A, where from the recurrence relation for the Bessel condition, the Schottky condition can be derived and proofed [18] [19] stating that:

\subsection{In the Case of $L$ (Axial Distance) $>R$ (Radial Distance)}

$$
\frac{D_{S}}{v_{i}}=\left(\frac{R}{2.405}\right)^{2}
$$

A proof of the Schottky condition will be discussed briefly by two methods in Appendix $\mathrm{A}$, where $(R \mathrm{~cm})$ represents the radial distance from the center to the edge through the cathode electrode measured by the Langmuir probe moved radially, and by substituting Equation (17) into (16), then

$$
\left(\frac{R}{2.4}\right)^{2}=\frac{k T_{e}}{m_{e} v_{e} v_{i}-\frac{m_{i} v_{i}^{2} n_{e}}{n_{+}}}
$$

Substituting the following equation into Equation (18)

$$
\begin{gathered}
v_{e}=N_{n}\left\langle Q_{e-n} v_{e-n}\right\rangle \\
v_{e}=\left(3.55 \times 10^{16} P\right)\left\langle Q_{e-n}\right\rangle\left(\frac{2 k T_{e}}{m_{e}}\right)^{1 / 2} \\
v_{i}=\left(3.55 \times 10^{16} P\right)\left\langle Q_{i-n}\right\rangle\left(\frac{2 k T_{i}}{m_{i}}\right)^{1 / 2}
\end{gathered}
$$

where $P$ in torr, $Q_{e-n}$ and $Q_{i-n}$ are the cross sections of electron-neutral and ion-neutral collisions, respectively results in

$$
\left(\frac{R}{2.4}\right)^{2}=\frac{k T_{e}}{\left[m_{e}\left(\frac{2 k T_{e}}{m_{e}}\right)^{1 / 2}\left(\frac{2 k T_{i}}{m_{i}}\right)^{1 / 2}\left(3.55 \times 10^{16} P\right)^{2}\left\langle Q_{i}\right\rangle\left\langle Q_{e}\right\rangle\right]-\left[\frac{n_{e}}{n_{+}} m_{i}\left[\left(3.55 \times 10^{16} P\right)^{2}\left\langle Q_{i}\right\rangle\right]^{2}\left(\frac{2 k T_{i}}{m_{i}}\right)\right]}(20)
$$


then

$$
\left(\frac{R P}{2.4}\right)^{2}=\frac{1}{\left[\left(\frac{4 m_{e} k T_{i}}{m_{i} k T_{e}}\right)^{1 / 2}\left(3.55 \times 10^{16}\right)^{2}\left\langle Q_{i}\right\rangle\left\langle Q_{e}\right\rangle\right]-\left[\frac{n_{e}}{n_{+}}\left(3.55 \times 10^{16}\left\langle Q_{i}\right\rangle\right)^{2}\left(\frac{2 k T_{i}}{m_{i} k T_{e}}\right)\right]}
$$

But from [20] [21] $\frac{\mu_{e}}{\mu_{i}} \cong 7.64\left(m_{i}\right)^{0.5}$

Or

$$
m_{i} \cong\left(\frac{1}{7.64} \frac{\mu_{e}}{\mu_{i}}\right)^{2}
$$

Then substituting (7) into (22a) gives

$$
\frac{1}{m_{i}} \cong\left(7.64 \frac{k T_{i}}{k T_{e}}\right)^{2}
$$

Moreover, substituting (22b), $\frac{m_{i}}{m_{e}} \cong 2000$ and $\frac{v_{i}}{v_{e}} \cong 10^{-2}$ into (21) gives

$$
\begin{aligned}
\left(\frac{R P}{2.4}\right)^{2} & =\frac{1}{\left[\left(7.64 \frac{k T_{i}}{k T_{e}}\right)\left(\frac{4 m_{e} k T_{i}}{k T_{e}}\right)^{1 / 2}\left(3.55 \times 10^{16}\right)^{2}\left\langle Q_{i}\right\rangle\left\langle Q_{e}\right\rangle\right]-\left[\frac{n_{e}}{n_{+}}\left(3.55 \times 10^{16}\left\langle Q_{i}\right\rangle\right)^{2}\left(7.64 \frac{k T_{i}}{k T_{e}}\right)^{2}\left(\frac{2 k T_{i}}{k T_{e}}\right)\right]} \\
(R P)^{2} & =\frac{2.4^{2}}{\left[\left(7.64 \frac{k T_{i}}{k T_{e}}\right)\left(\frac{4 m_{e} k T_{i}}{k T_{e}}\right)^{1 / 2}\left(3.55 \times 10^{16}\right)^{2}\left\langle Q_{i}\right\rangle\left\langle Q_{e}\right\rangle\right]-\left[\frac{n_{e}}{n_{+}}\left(3.55 \times 10^{16}\left\langle Q_{i}\right\rangle\right)^{2}\left(7.64 \frac{k T_{i}}{k T_{e}}\right)^{2}\left(\frac{2 k T_{i}}{k T_{e}}\right)\right]}
\end{aligned}
$$

Substituting the values of the cross sections, masses of ions and electrons into (24) gives

$$
(R P)^{2}=\frac{2.4^{2}}{(3.55)^{2}(7.64)(2)\left[\left[\left(\frac{k T_{i}}{k T_{e}}\right)\left(\frac{m_{e} k T_{i}}{k T_{e}}\right)^{1 / 2}\right]-\left[\frac{n_{e}}{n_{+}} 7.64\left(\frac{k T_{i}}{k T_{e}}\right)^{2}\left(\frac{k T_{i}}{k T_{e}}\right)\right]\right]}
$$

or

$$
(R P)^{2}=\frac{2.4^{2}}{(3.55)^{2}(7.64)(2)\left(\frac{k T_{i}}{k T_{e}}\right)^{3 / 2}\left[m_{e}^{1 / 2}-7.64 \frac{n_{e}}{n_{+}}\left(\frac{k T_{i}}{k T_{e}}\right)^{3 / 2}\right]}
$$

Then finally

$$
R P=0.1729 \frac{1}{\left(\frac{k T_{i}}{k T_{e}}\right)^{3 / 4}\left[m_{e}^{1 / 2}-7.64 \frac{n_{e}}{n_{+}}\left(\frac{k T_{i}}{k T_{e}}\right)^{3 / 2}\right]^{1 / 2}}
$$

Using the data of the electron temperatures and densities shown in Figure 4 
and Figure 5 respectively, into the Equation (27), taking into account that $T_{e} \gg T_{i} \quad\left(T_{i}=0.1 T_{e}\right), \frac{m_{e}}{m_{i}} \cong 2000$ [22] and for the spherical probe, the ion density is given by [23]:

$$
n_{+}=\frac{I_{+}}{0.6}\left(\frac{k T_{e}}{m_{i}}\right)^{1 / 2} A_{p}
$$

Knowing $T_{\mathrm{e}}\left(A_{p}=4 \pi r_{p}^{2}\right)$ area of spherical probe) and calculating positive ion current $I_{+}$from the I-V characteristic curve of the spherical single probe [24], $n_{+}$ can be determined.

Figure 7 shows the radial distribution of the electron temperature (RDFT) theoretically using Equation (27), in the abnormal cathode fall region from the center to the edge of the electrode as a function of $\mathrm{He}$ as an inert gas. The RDFT from the theoretical method presented above accurately shows a dramatic radial reduction of the electron temperature for any region (cathode fall or negative glow or positive column) and for any applied pressure. Furthermore, the decrements of $T_{e}$ at the edge of the electrode are more pronounced at the center due to edge effect [25].

\subsection{In the Case of $L$ (Axial Distance) $<R$ (Radial Distance)}

$$
\frac{D_{S}}{v_{i}}=\left(\frac{R}{2.405}\right)^{2}+\left(\frac{L}{\pi}\right)^{2}
$$

and by substituting Equation (29) into (16), then

$$
\left(\frac{R}{2.4}\right)^{2}+\left(\frac{L}{\pi}\right)^{2}=\frac{k T_{e}}{m_{e} v_{e} v_{i}-\frac{m_{i} v_{i}^{2} n_{e}}{n_{+}}}
$$

Substituting by Equations (19a, b and c) into Equation (30), respectively results in

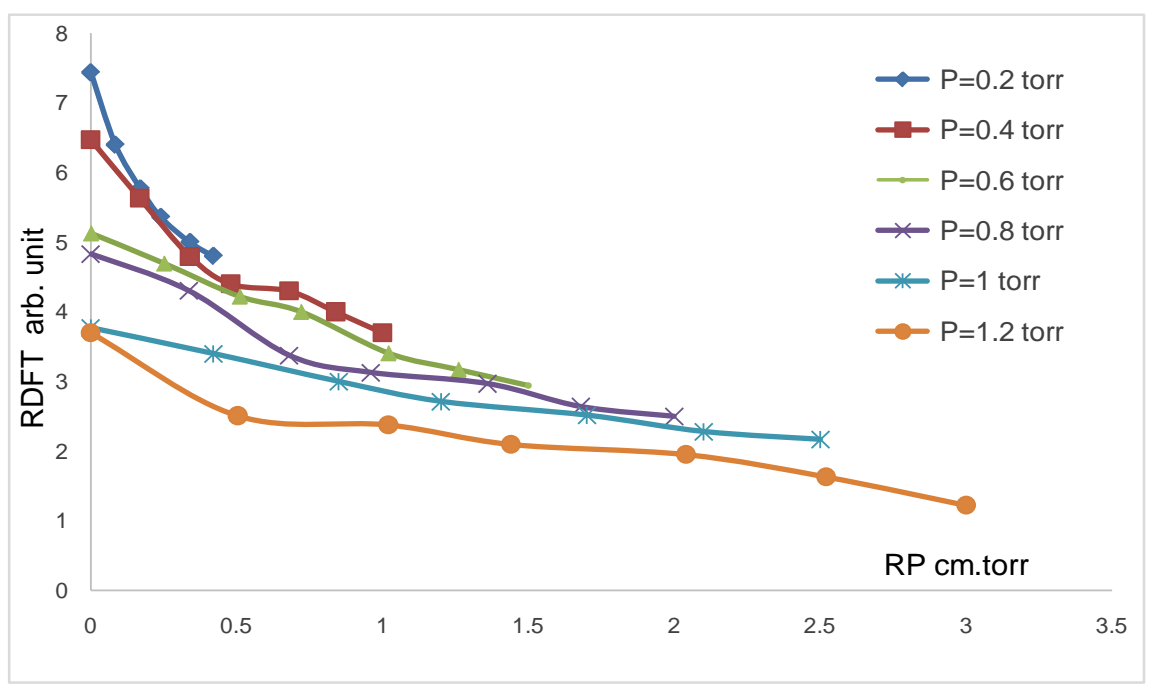

Figure 7. Theoretical radial distribution of the electron temperature (RDFT). 


$$
\begin{aligned}
& \left(\frac{R}{2.4}\right)^{2}+\left(\frac{L}{\pi}\right)^{2}=\frac{k T_{e}}{\left[m_{e}\left(\frac{2 k T_{e}}{m_{e}}\right)^{1 / 2}\left(\frac{2 k T_{i}}{m_{i}}\right)^{1 / 2}\left(3.55 \times 10^{16} P\right)^{2}\left\langle Q_{i}\right\rangle\left\langle Q_{e}\right\rangle\right]-\left[\frac{n_{e}}{n_{+}} m_{i}\left[\left(3.55 \times 10^{16} P\right)^{2}\left\langle Q_{i}\right\rangle\right]^{2}\left(\frac{2 k T_{i}}{m_{i}}\right)\right]} \\
& \text { then } \\
& \left(\frac{R}{2.4}\right)^{2}+\left(\frac{L}{\pi}\right)^{2}=\frac{1}{\left[\left(\frac{4 m_{e} k T_{i}}{m_{i} k T_{e}}\right)^{1 / 2}\left(3.55 \times 10^{16}\right)^{2}\left\langle Q_{i}\right\rangle\left\langle Q_{e}\right\rangle\right]-\left[\frac{n_{e}}{n_{+}}\left(3.55 \times 10^{16}\left\langle Q_{i}\right\rangle\right)^{2}\left(\frac{2 k T_{i}}{m_{i} k T_{e}}\right)\right]} \\
& \left(\frac{R P}{2.4}\right)^{2}+\left(\frac{L}{\pi}\right)^{2}=\frac{1}{\left[\left(7.64 \frac{k T_{i}}{k T_{e}}\right)\left(\frac{4 m_{e} k T_{i}}{k T_{e}}\right)^{1 / 2}\left(3.55 \times 10^{16}\right)^{2}\left\langle Q_{i}\right\rangle\left\langle Q_{e}\right\rangle\right]-\left[\frac{n_{e}}{n_{+}}\left(3.55 \times 10^{16}\left\langle Q_{i}\right\rangle\right)^{2}\left(7.64 \frac{k T_{i}}{k T_{e}}\right)^{2}\left(\frac{2 k T_{i}}{k T_{e}}\right)\right]} \\
& (R P)^{2}=\frac{2.4^{2}}{\left[\left(7.64 \frac{k T_{i}}{k T_{e}}\right)\left(\frac{4 m_{e} k T_{i}}{k T_{e}}\right)^{1 / 2}\left(3.55 \times 10^{16}\right)^{2}\left\langle Q_{i}\right\rangle\left\langle Q_{e}\right\rangle\right]-\left[\frac{n_{e}}{n_{+}}\left(3.55 \times 10^{16}\left\langle Q_{i}\right\rangle\right)^{2}\left(7.64 \frac{k T_{i}}{k T_{e}}\right)^{2}\left(\frac{2 k T_{i}}{k T_{e}}\right)\right]}-2.4^{2}\left(\frac{L}{\pi}\right)^{2}
\end{aligned}
$$

Substituting the values of the cross sections, masses of ions and electrons into (34) gives finally

$$
R P=\sqrt{\left(\frac{0.0299}{\left(\frac{k T_{i}}{k T_{e}}\right)^{3 / 2}\left[m_{e}^{1 / 2}-7.64 \frac{n_{e}}{n_{+}}\left(\frac{k T_{i}}{k T_{e}}\right)^{3 / 2}\right]}-5.76\left(\frac{L}{\pi}\right)^{2}\right)}
$$

For 1.2 torr of applied pressure, Figure 8 shows comparison between the radial distribution of the electron temperature (RDFT) for $L<R$ using Equation (35) and for $L>R$ using Equation (27), in the abnormal cathode fall region from the center to the edge of the electrode as a function of $\mathrm{He}$ as an inert gas.

The reasons for the radial distribution of the electron temperatures for both cases.

$L<R$ and $L>R$ are the following.

a) The temperature decreases for increasing pressure may be due to the relation between the temperature and the pressure given by [26]:

$$
\left|T_{e}\right|=6.5\left|P^{-0.066}\right|
$$

b) As the pressure increase leads to a further increase in the breakdown voltage, a sharp increase in electron density and a higher electron-electron collision frequency, leads to the decrease of the electron temperatures [27].

c) The present theoretical and experimental results are compatible and agree fairly well with the behavior of the data of von Engel [28] [29] [30] for the radial distribution of the electron temperature.

For $L<R$, RDFT accurately shows a dramatic decrease by $60 \%$ less than $R D F T$ values for $L>R$. This may be attributed to: 


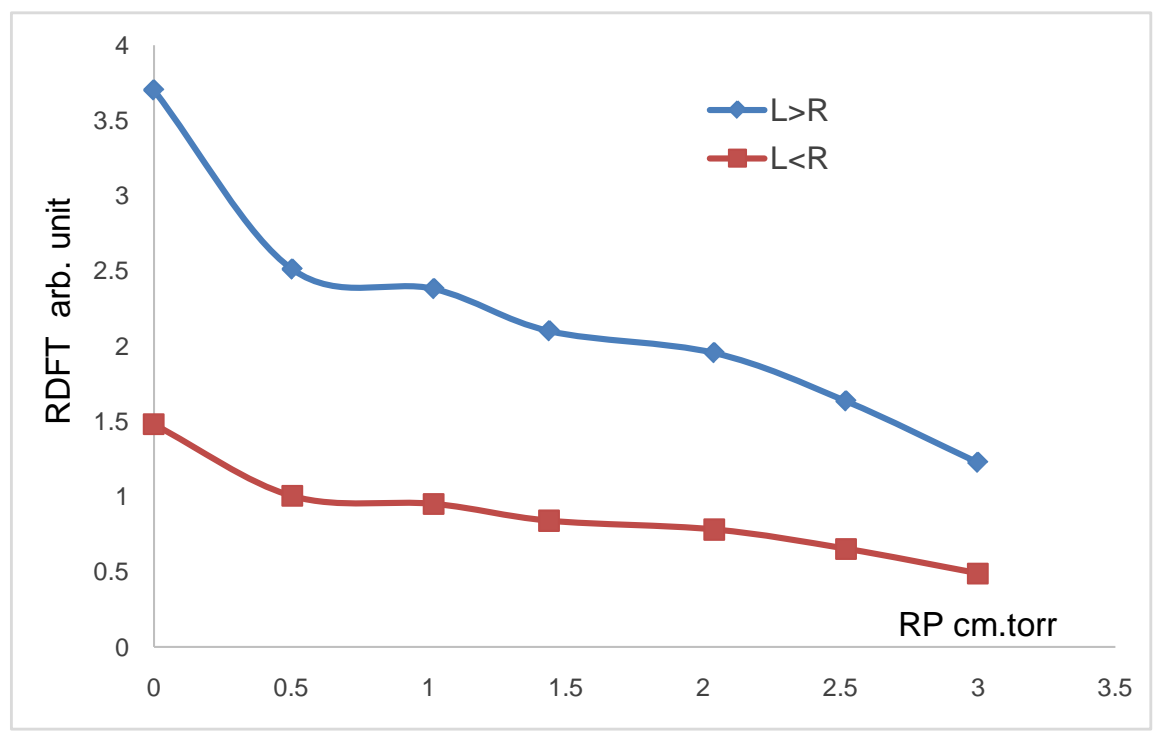

Figure 8. Comparison between the radial distribution of the electron temperature (RDFT) for $\mathrm{L}<\mathrm{R}$ and for $\mathrm{L}>\mathrm{R}$, at $\mathrm{P}=1.2$ torr.

The cathode fall, negative glow and positive column regions are compressed when $L<R$, i.e. the cathode fall region is compressed in thickness and henceforth higher potentials are expected. Thus, strong electric field is produced and therefore ions would accelerate, and more efficient sputtering processes take place. The produced plasma temperatures decrease, and densities increases; this means that the rate of plasma loss by diffusion decreased similarly as in the applied of magnetized DC plasma [23], therefore the current and current density are increased.

\section{Conclusion}

Theoretically, using the Einstein relation of ambipolar diffusion, charge density of the plasma, conductivity at the axis of the tube, the Schottky condition, and the cross sections of ion-neutral and electron-neutral collisions, the decrease of the radial distribution function of the electron temperature with increasing product of the radial distance and gas pressures can be determined. The theoretical study agrees well with experimental data of electron and ion temperatures and gives the radial decrement dependence of the electron temperatures from the center to the edge of the electrode.

\section{Acknowledgements}

GVO acknowledges for the partial financial support from MEPhI and NRU MPEI in the framework of the Russian Academic Excellence Project.

\section{Conflicts of Interest}

The authors declare no conflicts of interest regarding the publication of this paper. 


\section{References}

[1] Chen, F.F. (1965) Plasma Diagnostic Techniques. Academic Press, New York.

[2] Radmilovic-Radjenovic, M. and Radjenovic, B. (2006) Journal of Physics D: Applied Physics, 39, 3002. https://doi.org/10.1088/0022-3727/39/14/019

[3] Kagan, Y. and Paskalev, K.K. (1975) Soviet Physics. Technical Physics, 19, 1604.

[4] Kagan, Y. and Perel, V.I. (1964) Soviet Physics Uspekhi, 6, 767. https://doi.org/10.1070/PU1964v006n06ABEH003611

[5] Mott-Smith and Langmuir (1926) Physical Review, 28, 727. https://doi.org/10.1103/PhysRev.28.727

[6] Parent, B., Macheret, S.O. and Shneider, M.N. (2011) Journal of Computational Physics, 230, 8010-8027. https://doi.org/10.1016/j.jcp.2011.07.006

[7] Holt, E.H. and Haskell, R.E. (1965) Foundations of Plasma Dynamics. 2nd Edition, MacMillan Company, New-York.

[8] Huang, P.G., Shang, J.S. and Stanfield, S.A. (2011) AIAA Journal, 49, 119. https://doi.org/10.2514/1.J050463

[9] Bisek, N.J., Boyd, I.D. and Poggie (2009) Journal of Spacecraft and Rockets, 46, 568. https://doi.org/10.2514/1.39032

[10] Galaly, A.R. and Khedr, M.A. (2015) British Journal of Applied Science \& Technology, 11, 1-14. https://doi.org/10.9734/BJAST/2015/17663

[11] Galaly, A.R. (2016) British Journal of Applied Science \& Technology, 13, 1.

[12] Galaly, A.R. and El Akshar, F.F. (2013) Physica Scripta, 88, Article ID: 065503. https://doi.org/10.1088/0031-8949/88/06/065503

[13] Yasuda, H.K., Tao, W.H. and Prelas, M.A. (1996) Journal of Vacuum Science \& Technology A: Vacuum, Surfaces, and Films, 14, 2113. https://doi.org/10.1116/1.580089

[14] Nasser, E. (1971) Fundamentals of Gaseous Ionization and Plasma Electronics. Wiley-Interscience, New York.

[15] Raizer, Y.P. (1999) Gas Discharge Physics. Springer, New York.

[16] Von Engel, A.H. (1964) Ionized Gases. Oxford University Press, Oxford.

[17] Franklin, R.N. (1977) Plasma Phenomena in Gas Discharges. Oxford University Press, Oxford.

[18] Lieberman, M.A. and Lichtenberg, A.J. (1994) Principles of Plasma Discharges for Materials Processing. Wiley Interscience, New York.

[19] Braithwaite, N.St.J. (2000) Plasma Sources Science and Technology, 9, 517. https://doi.org/10.1088/0963-0252/9/4/307

[20] Piel, A. (2011) Plasma Physics. Springer, Berlin.

[21] Kolobov, V.I. (2013) Physics of Plasmas, 20, Article ID: 101610. https://doi.org/10.1063/1.4823472

[22] Fruchtman, A. (2009) Plasma Sources Science and Technology, 18, Article ID: 025033. https://doi.org/10.1088/0963-0252/18/2/025033

[23] Simon, A. (1955) Physical Review, 98, 317. https://doi.org/10.1103/PhysRev.98.317

[24] Galaly, A.R. (2014) Physical Science International Journal, 4, 930-939. https://doi.org/10.9734/PSIJ/2014/9604

[25] Hutchinson (1990) Principles of Plasma Diagnostics. Cambridge University Press, Cambridge. 
[26] Huba, J.D. (2011) Plasma Formulary. Naval Research Laboratory Report NRL/PU/ 6790-94-265.

[27] Druyvesteyn, M.J. and Penning, F.M. (1940) Reviews of Modern Physics, 12, 87. https://doi.org/10.1103/RevModPhys.12.87

[28] Von Engel, A. and Corrigan, S.J.B. (1958) Proceedings of the Physical Society, 72, 786. https://doi.org/10.1088/0370-1328/72/5/314

[29] Von Engel, A. and Cozens, J.R. (1965) International Journal of Electronics, 19, 61-68. https://doi.org/10.1080/00207216508937799

[30] Von Engel, A. (1956) Proceedings of the Physical Society, 59, 468.

[31] Bowman, F. (1958) Introduction to Bessel Conditions. Dover, New York.

[32] Korenev, B.G. (2002) Bessel Condition and Their Applications. CRC Press Company, Boca Raton, London, New York, Washington DC.

[33] Arfken, G. (1985) Bessel Conditions. In: Mathematical Methods for Physicists, 3rd Edition, Academic Press, Orlando, Ch. 11, 573-636.

https://doi.org/10.1016/B978-0-12-059820-5.50019-7

[34] Gray, A. and Mathews, G.B. (1966) A Treatise on Bessel Conditions and Their Applications to Physics. 2nd Edition, Dover, New York.

[35] Watson, G.N. (1966) A Treatise on the Theory of Bessel conditions. 2nd Edition, Cambridge University Press, Cambridge. 


\section{Appendix: Proofing of the Schottky Condition}

There are two classes of Schottky condition models using.

Appendix A: the recurrence relation for the Bessel condition

Appendix B: the boundary conditions

$$
\frac{D_{S}}{v_{i}}=\frac{K T_{e}}{\frac{m_{i} v_{i}^{2} n_{e}}{n_{+}}+m_{e} v_{e} v_{i}}
$$

Due to the diffusion process during plasma formation an interesting process occur in the plasma formation stage of the basil discharge. The Schottky condition stating that

$$
\frac{D_{S}}{v_{i}}=\left(\frac{R}{2.4}\right)^{2} \text { can be demonstrated as follows: }
$$

Appendix A: From the Recurrence Relation for Bessel Condition [31] [32] [33]

$$
2 J_{n}^{\prime}(x)=J_{n-1}(x)-J_{n+1}(x)
$$

and

$$
\frac{2 n}{x} J_{n}(x)=J_{n-1}(x)+J_{n+1}(x)
$$

Subtracting equation (37) from (38), gives

$$
\frac{2 n}{x} J_{n}(x)-2 J_{n}^{\prime}(x)=J_{n-1}(x)+J_{n+1}(x)-J_{n-1}(x)+J_{n+1}(x)
$$

Or

$$
\frac{n}{x} J_{n}(x)-J_{n}^{\prime}(x)=J_{n+1}(x)
$$

Or

$$
n J_{n}(x)-x J_{n}^{\prime}(x)=x J_{n+1}(x)
$$

This results in

$$
x J_{n}^{\prime}(x)=n J_{n}(x)-x J_{n+1}(x)
$$

Differentiating (40) with respect to $x$, gives:

$$
x J_{n}^{\prime \prime}(x)+J_{n}^{\prime}(x)=n J_{n}^{\prime}(x)-x J_{n+1}^{\prime}(x)-J_{n+1}(x)
$$

Multiplying Equation (40) in $n / x$ gives:

$$
n J_{n}^{\prime}(x)=\frac{n^{2}}{x} J_{n}(x)-n J_{n+1}(x)
$$

Adding Equations (37) and (38) gives

$$
x J_{n}^{\prime}(x)=x J_{n-1}(x)-n J_{n}(x)
$$

Putting $n=n+1$ in Equation (43) gives 


$$
x J_{n+1}^{\prime}(x)=x J_{n}(x)-(n+1) J_{n+1}(x)
$$

Substituting (39) and (40) into (38), results in

$$
x J_{n}^{\prime \prime}(x)+J_{n}^{\prime}(x)=\frac{n^{2}}{x} J_{n}(x)-x J_{n}(x)
$$

Then

$$
J_{n}^{\prime \prime}(x)+\frac{1}{x} J_{n}^{\prime}(x)+\left(1-\frac{n^{2}}{x^{2}}\right) J_{n}(x)=0
$$

Finally

$$
J^{\prime \prime}+\frac{1}{x} J^{\prime}+\left(\lambda-\frac{n^{2}}{x^{2}}\right) J=0
$$

For $n=0$

$$
J^{\prime \prime}+\frac{1}{x} J^{\prime}+(\lambda) J=0
$$

For the Bessel condition in cylindrical coordinate

$$
x=K r=\left(\frac{1}{D_{a} \tau}\right)^{1 / 2} R=\left(\frac{\lambda}{D_{a}}\right)^{1 / 2} R
$$

with the solution $J_{n}(x)=\sqrt{\frac{2}{\pi}} \cos \left(x-\frac{n \pi}{2}-\frac{\pi}{4}\right)$, and $J_{0}=0$ for $n=0$, gives

$$
\begin{aligned}
& 0=\cos \left(x-\frac{\pi}{4}\right) \text { with the solution } x=\frac{3 \pi}{4} \approx 2.4 \\
& x=K r=\frac{R}{(D \tau)^{1 / 2}} \approx 2.4 \text { or } \frac{R}{2.4}=(D \tau)^{1 / 2}
\end{aligned}
$$

Then

$$
\frac{R}{2.4}=\left(\frac{D_{S}}{v_{i}}\right)^{1 / 2}
$$

or

$$
\frac{D_{S}}{v_{i}}=\left(\frac{R}{2.4}\right)^{2}
$$

Appendix B: From the Boundary Conditions [34] [35]

$$
n_{e} v_{e}=-D_{S} \nabla n_{e}
$$

Then

$$
D_{S}=-\frac{n_{e} v_{e}}{\nabla n_{e}}
$$

But

$$
v_{i}=\frac{\nabla \cdot n_{e} v_{e}}{n_{e}}
$$


Then

$$
\frac{D_{S}}{v_{i}}=-\left(\frac{n_{e}}{\nabla n_{e}}\right)^{2}
$$

For boundary conditions $r=R, n_{e}=n_{e 0}$, and $J_{0}=2.4$, then

$$
\nabla n_{e}=-\frac{2.4 n_{e}}{R}
$$

Substituting from (50) into (49) then we get

$$
\frac{D_{S}}{v_{i}}=\left(\frac{R}{2.4}\right)^{2}
$$

